\title{
Quantum trajectory analysis for electrical detection of single-electron spin resonance
}

\author{
Jinshuang Jin, Jianhong Guo, JunYan Luo, and Xin-Qi Li \\ State Key Laboratory for Superlattices and Microstructures, Institute of Semiconductors, Chinese Academy of Sciences, \\ P.O. Box 912, Beijing 100083, China \\ YiJing Yan \\ Department of Chemistry, Hong Kong University of Science and Technology, Kowloon, Hong Kong \\ (Received 9 September 2005; revised manuscript received 3 January 2006; published 13 March 2006)
}

\begin{abstract}
A Monte Carlo simulation on the basis of quantum trajectory approach is carried out for the measurement dynamics of a single-electron spin resonance. The measured electron, which is confined in either a quantum dot or a defect trap, is tunnel coupled to a side reservoir and continuously monitored by a mesoscopic detector. The simulation not only recovers the observed telegraphic signal of detector current, but also predicts unique features in the output power spectrum which are associated with electron dynamics in different regimes.
\end{abstract}

DOI: 10.1103/PhysRevB.73.125312 PACS number(s): 73.63.-b, 76.30.-v, 03.67.Lx, 85.35.-p

\section{INTRODUCTION}

In recent years, largely being stimulated by the interest of solid-state quantum computation, measurement of singleelectron spin has become an intensive research subject. The main difficulty of spin measurement lies in the inherent weakness of magnetic interaction, making its direct detection quite challenging. The current state-of-the-art techniques include, for instance, scanning tunneling microscopy, ${ }^{1}$ magnetic resonance force microscopy ${ }^{2}$ (MRFM), submicron Hall magnetometer, ${ }^{3}$ and spin-to-charge conversion, ${ }^{4}$ etc. Experimentally, detection of single-electron spin has been illustrated by optical means, ${ }^{5,6}$ MRFM technique, ${ }^{7}$ and electrical methods. ${ }^{8,9}$ In particular, the idea of electrical approach is based on the spin-to-charge conversion, which maps the spin states onto detectable charge states. In Refs. 8 and 9, the single shot readout of the charge state is demonstrated in terms of single realization of continuous measurement. In this work, we present a Monte Carlo simulation for the dynamics of continuous electrical measurement and calculate the output noise spectrum. This study provides a fundamental description for the stochastic nature of the state evolution, which stems from the interplay of the manipulation of coherent field and the (simultaneous) influence of incoherent reservoir. Also, the predicted unique features in the noise spectrum manifest distinct electron dynamics in different regimes.

In close connection with the experiments, ${ }^{8,9}$ we consider a model system of quantum dot in the Coulomb blockage regime, which is tunnel coupled to a side electronic reservoir and continuously monitored by an electrical detector, say, the quantum point contact (QPC), as is schematically shown in Fig. 1. The single-electron level in the dot is split by an external magnetic field $B_{0}$, i.e., with the Zeeman energy of $\epsilon_{\downarrow}-\epsilon_{\uparrow}=g \mu_{B} B_{0} \equiv \Delta$, where $g$ is the Landé- $g$ factor of electron spin and $\mu_{B}$ is the Bohr magneton. The spin-up and spindown states are coupled by an oscillating magnetic field $B_{\mathrm{ESR}}=B_{1} \cos (\omega t)$, applied perpendicularly to the field $B_{0}$, which leads to the well-known electron spin resonance (ESR) with Rabi frequency $w_{R}=g \mu_{B} B_{1}$. In the Coulomb blockade regime, the dot is occupied by at most one electron. Thus, the dot electron Hamiltonian in the presence of magnetic fields reads $H_{\mathrm{dot}}=(\Delta / 2)\left(d_{\downarrow}^{\dagger} d_{\downarrow}-d_{\uparrow}^{\dagger} d_{\uparrow}\right)+\left(\omega_{R} / 2\right)\left(d_{\downarrow}^{\dagger} d_{\uparrow} e^{-i \omega t}\right.$ $\left.+d_{\uparrow}^{\dagger} d_{\downarrow} e^{i \omega t}\right)$, in which the first part describes the Zeeman splitting, and the second one represents the electron spin resonance. Following the QPC measurement model proposed by Gurvitz, ${ }^{10}$ the entire measurement setup shown in Fig. 1 is described by the Hamiltonian $H=H_{0}+H_{T}+H_{\text {int }}$, with

$$
\begin{gathered}
H_{0}=H_{\mathrm{dot}}+H_{\mathrm{res}}+H_{\mathrm{PC}}, \\
H_{T}=\sum_{q \sigma}\left[t_{q} c_{q \sigma}^{\dagger} d_{\sigma}+\text { H.c. }\right], \\
H_{\mathrm{int}}=\sum_{k, q}\left(T_{q k}+\chi_{q k} n_{0}\right) a_{L k}^{\dagger} a_{R q}+\text { H.c. }
\end{gathered}
$$

Here the Hamiltonian $H_{\mathrm{res}}=\Sigma_{q \sigma} \epsilon_{q} c_{q \sigma}^{\dagger} c_{q \sigma}$ stands for the side electronic reservoir, and $H_{T}$ describes its tunnel coupling to

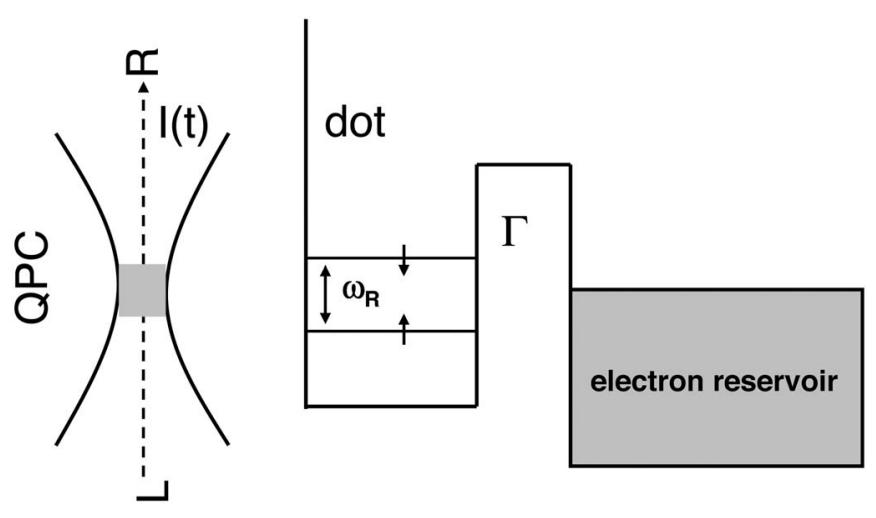

FIG. 1. Schematic diagram of a quantum dot being tunnel coupled to a side reservoir and measured by a quantum point contact (QPC). The single-electron level in the dot is split by an external magnetic field and the two spin states are coupled by an electron spin resonance (ESR) magnetic field. The tunneling of single electron into and out of the dot would alter the transport current through the QPC detector. 
the quantum dot. $\sigma=\uparrow, \downarrow$ denote the two possible orientations of electron spin. For the QPC detector, $H_{\mathrm{PC}}$ $=\sum_{k} \epsilon_{k}^{L} a_{L k}^{\dagger} a_{L k}+\sum_{q} \epsilon_{q}^{R} a_{R q}^{\dagger} a_{R q}$ stands for the two (left and right) reservoirs, and $H_{\text {int }}$ describes tunneling between them. Notice that the tunneling amplitude explicitly depends on the electron occupation of the quantum dot, which is modeled by $T_{q k}+\chi_{q k} n_{0}$, where $n_{0} \equiv 1-\Sigma_{\sigma} d_{\sigma}^{\dagger} d_{\sigma}$ is the vacancy operator of the quantum dot.

\section{REDUCED DESCRIPTION FOR THE MEASUREMENT PROCESS}

A significant aspect of quantum measurement is to investigate the back action of measurement device on the measured system, which results in the dephasing and relaxation. Technically, this can be realized by tracing out the microscopic degrees of freedom of the measurement device. Physically, this reduced description corresponds to ensemble results, i.e., all the measurement records are averaged. For present setup, the side reservoir and the detector can be treated as two independent environments acting on the (measured) quantum dot. As a model description, we assume the tunneling amplitude $T_{k q}$ to be real and independent of the reservoir state " $k q$," i.e., $H_{\text {int }}=Q F$, where $Q=T+\chi n_{0}$ and $F=\Sigma_{k q}\left(a_{L k}^{\dagger} a_{R q}+\right.$ H.c. $)$. For the coupling between the dot and the side reservoir, we reexpress the coupling Hamiltonian as $H_{T}=\Sigma_{\sigma}\left(d_{\sigma} f_{\sigma}^{\dagger}+\right.$ H.c. $)$, where $f_{\sigma}^{\dagger} \equiv \Sigma_{q} t_{q} c_{q \sigma}^{\dagger}$. By treating $H_{T}$ and $H_{\text {int }}$ as perturbation and on the basis of the second-order cummulant expansion, an equation for the reduced density matrix can be derived as ${ }^{11,12}$

$$
\begin{aligned}
\dot{\rho}= & -i \mathcal{L}_{0} \rho-\frac{1}{2}\left[Q, \widetilde{Q} \rho-\rho \widetilde{Q}^{+}\right]-\frac{1}{2} \sum_{\sigma}\left\{\left[d_{\sigma}^{\dagger}, D_{\sigma}^{(-)} \rho-\rho D_{\sigma}^{(+)}\right]\right. \\
& + \text {H.c. }\} .
\end{aligned}
$$

Here, the first term $\mathcal{L}_{0} \rho \equiv\left[H_{\mathrm{dot}}, \rho\right]$ describes the coherent manipulation of the dot electron (by the ESR magnetic field), while the second and third terms stem from the back action of the detector and the random tunneling caused by the side reservoir. In the back-action term, the operator $\widetilde{Q}=\widetilde{Q}^{(+)}+\widetilde{Q}^{(-)}$, and $\widetilde{Q}^{( \pm)}=C^{( \pm)}\left(\mathcal{L}_{0}\right) Q$, where the superoperators $C^{( \pm)}\left(\mathcal{L}_{0}\right)$ are the Fourier transform of the correlation functions $C^{(+)}(t)=\langle F(t) F(0)\rangle$ and $C^{(-)}(t)=\langle F(0) F(t)\rangle$. Under the wide-band approximation, $C^{( \pm)}\left(\mathcal{L}_{0}\right)$ can be explicitly carried out as: ${ }^{11} C^{( \pm)}\left(\mathcal{L}_{0}\right)=\eta\left[x / 1-e^{-\beta x}\right]_{x=-\left(\mathcal{L}_{0} \pm e V\right)}$. Here, $\eta=2 \pi g_{l} g_{r}$, with $g_{l}$ and $g_{r}$ as the energy-independent density of states for the two reservoirs of the QPC detector. $\beta=1 /\left(k_{B} T\right)$ is the inverse temperature, and $e V=\mu_{L}-\mu_{R}$ is the applied voltage across the detector. Similarly, for the side reservoir, we obtain: ${ }^{12} D_{\sigma}^{( \pm)}=\Gamma_{\sigma} n_{\sigma}^{( \pm)}\left(\epsilon_{\sigma}\right) d_{\sigma}$. Here $n_{\sigma}^{(+)}\left(\epsilon_{\sigma}\right)=n_{\sigma}\left(\epsilon_{\sigma}\right)$ and $n_{\sigma}^{(-)}\left(\epsilon_{\sigma}\right)=1-n_{\sigma}\left(\epsilon_{\sigma}\right)$, with $n_{\sigma}$ the Fermi distribution function. $\Gamma_{\sigma}=2 \pi g_{c}\left|t_{q}\right|^{2}$, in which $g_{c}$ is the density of states of the side reservoir electrons at the energy $\epsilon_{\sigma}$.

At the limit of large bias voltage, ${ }^{10,13}$ Eq. (2) can be further simplified to the Lindblad-type master equation

$$
\dot{\rho}=-i \mathcal{L}_{0} \rho+\Gamma_{\downarrow} \mathcal{D}\left[S_{1}\right] \rho+\Gamma_{\uparrow} \mathcal{D}\left[S_{2}\right] \rho+\mathcal{D}\left[\mathcal{T}+\mathcal{X} n_{0}\right] \rho \equiv \mathcal{L} \rho(t)
$$

Here, we have also restricted our study to zero temperature. The superoperator $\mathcal{D}$ is defined as $\mathcal{D}[r] \rho=\mathcal{J}[r] \rho-\mathcal{A}[r] \rho$, where $\mathcal{J}[r] \rho \equiv r \rho r^{\dagger}$, and $\mathcal{A}[r] \rho \equiv \frac{1}{2}\left(r^{\dagger} r \rho+\rho r^{\dagger} r\right)$. The jump operators $S_{1} \equiv|0\rangle\langle\downarrow|$ and $S_{2} \equiv|\uparrow\rangle\langle 0|$ describe the tunneling between the dot and the side reservoir. The homodyne-type jump operator $\mathcal{D}\left[\mathcal{T}+\mathcal{X} n_{0}\right]$, which describes the back action of the detector, is associated with the tunneling amplitudes through the QPC with and without an electron in the quantum dot, by the corresponding tunneling rates $|\mathcal{T}|^{2}=\eta|T|^{2} \mathrm{eV}$ $\equiv D$, and $|\mathcal{T}+\mathcal{X}|^{2}=\eta|T+\chi|^{2} e V \equiv D^{\prime}$. Note that, differing from the setup of a charge qubit measured ${ }^{10}$ by a QPC, here the QPC's back action has minor effect on the measured-electron dynamics. The reason is that, in the occupation state basis of the quantum dot, $\mathcal{D}\left[\mathcal{T}+\mathcal{X} n_{0}\right] \rho$ only contributes to the offdiagonal elements, which are decoupled from the diagonal ones. That is, the back action only causes dephasing between the "occupied" and "empty" dot states. But no phase coherence is introduced initially and neither will it be generated in the later evolution. Thus, the QPC has no influence on the electron dynamics under study. ${ }^{14}$

Straightforwardly, the (ensemble) measurement current through the detector can be calculated via

$$
I=I_{0} \rho_{00}+I_{1} \rho_{11},
$$

where $I_{0}=e D^{\prime}$ and $I_{1}=e D$ are, respectively, the currents through the QPC in the absence and presence of an electron in the quantum dot. Note that Eq. (4) is precisely the result presented in Ref. 15. In the following, we will see that it is indeed an ensemble average of the continuously measured currents from a large number of individual realizations.

\section{QUANTUM TRAJECTORY DESCRIPTION}

The above reduced density matrix description corresponds to average over all the measurement records of many individual realizations. However, in most cases, the original readout data of continuous measurement of a single quantum system is the record from a single measurement realization. For instance, the experimental result of Ref. 9 reveals a random telegraph signal (RTS), with a detector current jumping between two discrete values stochastically.

Proper description for this single measurement realization is the quantum trajectory theory developed in quantum optics, ${ }^{16,17}$ which describes the system evolution conditioned on the information continuously acquired by the detector. The central ingredient of this formalism is the conditional master equation (CME), which is an unraveling of the unconditional master equation, Eq. (3).

For the tunneling events between the quantum dot and the side reservoir, two stochastic point variables $d N_{1}(t)$ and $d N_{2}(t)$ (with values either 0 or 1 ) are employed to denote, respectively, the electron numbers that tunnel from the dot to the side reservoir and vice versa, during the infinitesimal time interval $d t$. While for the QPC, at the limit of zero temperature and large bias voltage, the electron only tunnels 
from the source (left reservoir) to the drain (right reservoir), which is denoted by an alternative stochastic variable $d N_{c}(t)$. Following Ref. 13, we obtain

$$
\begin{aligned}
d \rho_{c}(t)= & d t\left\{-i \mathcal{L}_{0}-\mathcal{A}\left[\mathcal{T}+\mathcal{X}_{0}\right]-\Gamma_{\downarrow} \mathcal{A}\left[S_{1}\right]-\Gamma_{\uparrow} \mathcal{A}\left[S_{2}\right]+\mathcal{P}_{c}(t)\right. \\
& \left.+\mathcal{P}_{1}(t)+\mathcal{P}_{2}(t)\right\} \rho_{c}(t)+d N_{1}(t)\left[\frac{\Gamma_{\downarrow} \mathcal{J}\left[S_{1}\right]}{\mathcal{P}_{1}(t)}-1\right] \rho_{c}(t) \\
& +d N_{2}(t)\left[\frac{\Gamma_{\uparrow} \mathcal{J}\left[S_{2}\right]}{\mathcal{P}_{2}(t)}-1\right] \rho_{c}(t) \\
& +d N_{c}(t)\left[\frac{\left.\mathcal{J} \mathcal{T}+\mathcal{X} n_{0}\right]}{\mathcal{P}_{c}(t)}-1\right] \rho_{c}(t)
\end{aligned}
$$

in which the stochastic variables $d N_{i}(t)(i=c, 1,2)$ have the ensemble property $\left.E\left[d N_{i}(t)\right]=\mathcal{P}_{i}(t) d t=\operatorname{Tr}\left\{\mathcal{J} r_{i}\right] \rho_{c}(t)\right\} d t$, with $r_{c}=\mathcal{T}+\mathcal{X} n_{0}, r_{1}=\sqrt{\Gamma_{\downarrow}} S_{1}$, and $r_{2}=\sqrt{\Gamma_{\uparrow}} S_{2}$, respectively. Here $E[\cdots]$ denotes an ensemble average of a large number of stochastic processes.

Within the quantum trajectory approach, the current through the QPC is given by

$$
I(t)=e d N_{c}(t) / d t .
$$

Here, we show that its ensemble average recovers the current given by Eq. (4). With the help of the CME, Eq. (5), the stationary-state current can be evaluated as

$$
\begin{aligned}
I_{\infty} & =e E\left[d N_{c}(t) / d t\right]_{t \rightarrow \infty} \\
& \left.=e \operatorname{Tr}\left\{\mathcal{J} \mathcal{T}+\mathcal{X} n_{0}\right] \rho(\infty)\right\} \\
& =e\left[D+\left(D^{\prime}-D\right) \frac{\omega_{R}^{2}}{\Gamma^{2}+3 \omega_{R}^{2}+4 \delta^{2}}\right],
\end{aligned}
$$

where $\rho(\infty)$ is the ensemble stationary state calculated by Eq. (3). Here, we have assumed $\Gamma_{\uparrow}=\Gamma_{\downarrow} \equiv \Gamma$. We notice that Eq. (7) is precisely the same as derived in Ref. 15 based on Eq. (4).

For the typical mesoscopic detector QPC, the condition $D \gg\left|D^{\prime}-D\right|$ implies a large background current. Accordingly, it is desirable to convert the above point process description into a diffusive one. ${ }^{13}$ First, the terms in Eq. (5), $\quad\left\{-\mathcal{A}\left[\mathcal{T}+\mathcal{X} n_{0}\right]+\mathcal{P}_{c}(t)\right\} \rho_{c}(t) d t+d N_{c}(t)\left[\mathcal{J}\left[\mathcal{T}+\mathcal{X} n_{0}\right] /\right.$ $\left.\mathcal{P}_{c}(t)-1\right] \rho_{c}(t)$, are replaced by $\mathcal{D}\left[\mathcal{T}+\mathcal{X} n_{0}\right] \rho_{c}(t) d t+\mathcal{X}\left[n_{0} \rho_{c}(t)\right.$ $\left.+\rho_{c}(t) n_{0}-2\left\langle n_{0}\right\rangle \rho_{c}(t)\right] \xi(t) d t$, where $\xi(t)$ is a Gaussian white noise characterized by $E[\xi(t)]=0$ and $E\left[\xi\left(t^{\prime}\right) \xi(t)\right]=\delta\left(t-t^{\prime}\right)$. Then, the detector current $I(t)$ of Eq. (6) in the diffusive regime becomes

$$
I(t)-\bar{I}=\Delta I\left[\rho_{00}^{c}(t)-\rho_{11}^{c}(t)\right] / 2+\sqrt{S_{0} / 2} \xi(t),
$$

where $\bar{I}=\left(I_{0}+I_{1}\right) / 2, \Delta I=I_{0}-I_{1}$, and the background shot noise $S_{0}=2 e I_{\infty}$. Here, we introduced the notation $\rho_{i j}^{c}(t)$ $\equiv\left\langle i\left|\rho_{c}\right| j\right\rangle$, with " $(i, j)=(0,1)$ " characterizing the number of electrons occupied in the dot.

From Eq. (8), we find that the detector current $I(t)$ basically follows the occupation probabilities $\rho_{i i}^{c}(t)$ and contains a noise term $\xi(t)$ that purely arises from the intrinsic fluctuations in the detector, being independent of the measured system. Particularly, in the Markov limit, the detector noise sub-

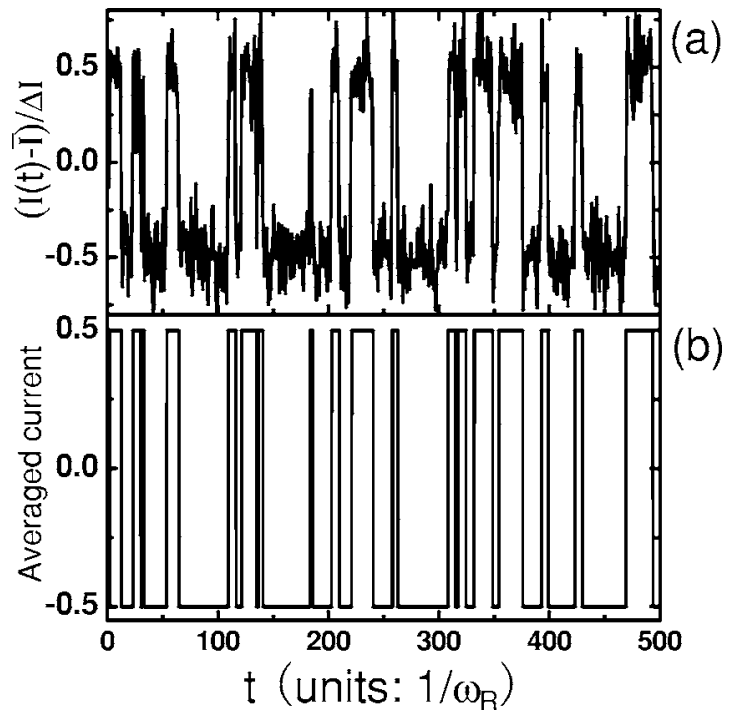

FIG. 2. Detector current from a single particular realization of quantum measurement, where the feature of random telegraph signal (RTS) which jumps between two discrete current values is evident. In the simulation, the resonance condition (i.e., $\delta=\Delta-\omega=0$ ) and weak coupling between the dot and the side reservoir (by setting $\left.\Gamma=0.1 \omega_{R}\right)$ are assumed. The simulated random current $I(t)$ in (a) is filtered by using a rectangular window with width $\tau=0.9 / \omega_{R}$, and in (b) is further averaged over the detector's background current fluctuations.

jects to Gaussian distribution as characterized above. For noise strength weaker than the signal, single particular realization of measurement will be evident. Since the fluctuation grows when $I(t)$ is examined at smaller time scales, proper averaging over time ("low-pass filtering") is necessary. ${ }^{18}$ Based on Eq. (8), we thus average $I(t)$ by using a rectangular filter window with time width $\tau, I(t)=\tau^{-1} \int_{t-\tau}^{t} I\left(t^{\prime}\right) d t^{\prime}$. The width of the filtering window should be larger than the average time interval of two successive electron tunneling events through the QPC junction and smaller than the average occupied/unoccupied time of the quantum dot. This makes the output current not too noisy in order to extract the information of the measured system. The numerical result is shown in Fig. 2(a), where the width of the filtering window is chosen as $\tau=0.9 / \omega_{R}$. As a manifestation of the telegraph signal, the current stochastically switches between two discrete values. The low and high current plateaus correspond to the quantum dot with and without an extra electron in it. The average widths of the plateaus are determined by the average occupation probabilities $\rho_{i i}^{c}(t)$. Also, the detector shot noise would cause current fluctuations around the plateaus. If the detector's background current fluctuations are further averaged, the telegraph signal will be more evident, as shown in Fig. 2(b), which agrees well with the experimental observation reported in Ref. 9, in the regime $\Gamma \ll \omega_{R}$.

As we have seen in Fig. 2, the single realization of the output current largely reflects the instantaneous occupation of the quantum dot, which is determined by the interplay of the coherent ESR driving and the incoherent tunneling. Specifically, we can easily imagine: (i) in the weak tunneling regime, the dot is either occupied or unoccupied for a long 

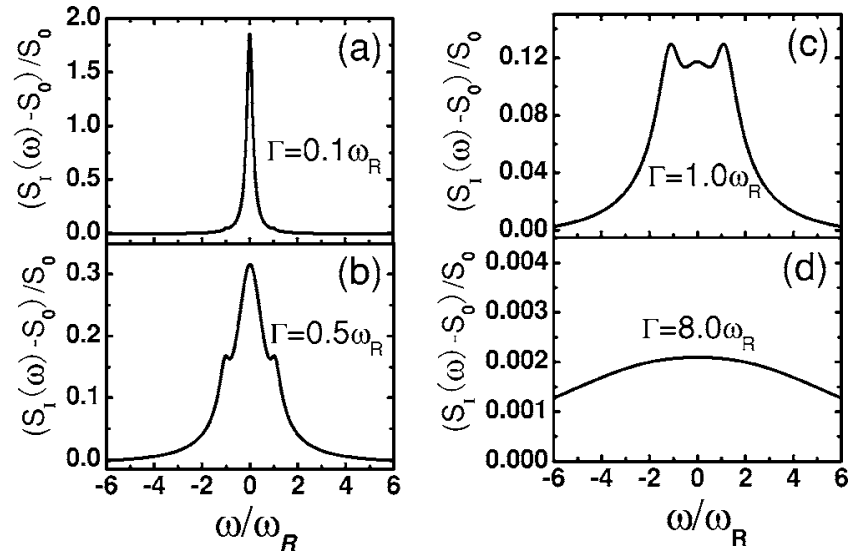

FIG. 3. Stationary noise power spectrum of the detector current. Panels (a)-(d) correspond to different coupling strengths $\Gamma$ between the quantum dot and the side reservoir.

time on average; (ii) in the intermediate tunneling regime, the dot is occupied for longer time than unoccupied; and (iii) in the strong tunneling regime, the dot is largely occupied. Accordingly, the output current would follow these occupations and exhibit the corresponding behaviors. Rather than showing the entire landscape of the dot-electron dynamics by the output current, in the following, we show that it can be elegantly manifested in the output power spectrum.

\section{NOISE SPECTRUM}

Following Refs. 19 and 20, as deriving the ensembleaveraged current Eq. (7), starting with the current formula Eq. (6) which is defined in the "point process" regime can also lead to the current correlation function in the stationary state as

$$
\begin{aligned}
G(\tau)= & \{E[I(t+\tau) I(t)]-E[I(t+\tau)] E[I(t)]\}_{t \rightarrow \infty} \\
= & e I_{\infty} \delta(\tau)+e^{2}\left(D^{\prime}-D\right)^{2} \\
& \times\left\{\operatorname{Tr}\left[n_{0} e^{\mathcal{L} \tau} \mathcal{J}\left[n_{0}\right] \rho_{\infty}\right]-\operatorname{Tr}\left[n_{0} \rho_{\infty}\right]^{2}\right\} .
\end{aligned}
$$

It should be noted that although this expression was derived in the point process regime, it is still applicable in the diffusive regime, since it is an ensemble averaged result. By noting $G(-\tau)=G(\tau)$, the stationary noise power spectrum can be computed by

$$
S(w)=2 \int_{-\infty}^{\infty} d \tau G(\tau) e^{-i w \tau} .
$$

Numerical results of the noise spectrum are shown in Fig. 3, where the dot-electron dynamics under the interplay of the coherent ESR driving and the incoherent tunneling is clearly manifested. In the weak tunneling regime (i.e., $\Gamma \ll \omega_{R}$ ), compared to the electron spin-up and spin-down flipping time in the dot, the electron tunnels into and out of the dot slowly. As a consequence, the dot occupation only changes occasionally with long-time separation, which results in the dominant sharp Lorentzian peak around the zero frequency, see Fig. 3(a). This feature indicates nothing but the telegraph noise.

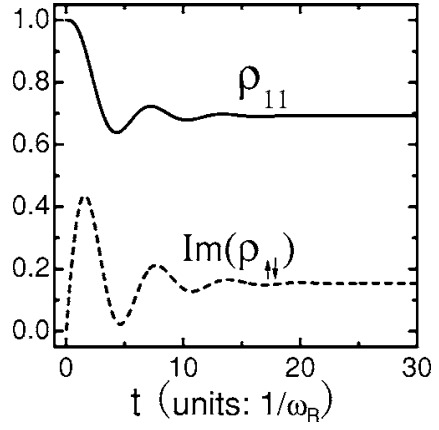

FIG. 4. ESR-induced coherent oscillation of the electron occupation probability in the quantum dot (solid curve). The nondiagonal element $\rho_{\uparrow \downarrow}$ describes the phase coherence of the ESR (dashed curve), where the decay is caused by the tunnel coupling with the side reservoir (but not the back action of the QPC detector). The adopted parameters are the same as for Fig. 3(b).

With the increase of $\Gamma$, the in-dot coherent flipping will eventually play an important role in the electron's dynamics, which leads to the gradual suppression of the central sharp peak (i.e., the telegraph noise spectrum), and makes the coherent peak around the Rabi frequency more evident, as shown by Figs. 3(b) and 3(c). Finally, as $\Gamma \gg \omega_{R}$, the incoherent tunneling between the dot and the side reservoir takes place so fast that the dot is almost occupied for all time, which greatly reduces the excess noise as shown in Fig. 3(d). The considerably flattened peak shown there simply reflects the sudden change of the dot occupation, which resembles the white noise process.

The above behaviors of the noise spectrum are closely related to the output current characteristics in different regimes, as discussed previously in the context of Fig. 2. Below, we further discuss the coherent peaks in Fig. 3: (i) We would like to point out that the parameters $D$ and $D^{\prime}$ are related to the background noise $S_{0}=2 e I_{\infty}$ [the first term of Eq. (9)], meanwhile $D-D^{\prime}$ would scale the magnitude of the excess noise which is caused by the electron dynamics in the quantum dot [see the second term of Eq. (9)]. However, $D$ and $D^{\prime}$ have no effect on the intrinsic structure of the noise spectrum. (ii) The height of the coherent peak in the noise spectrum is not very sensitive to the tunneling rate $\Gamma$, but its evolution from "birth" to "death" by increasing $\Gamma$ is evident. Instead of its absolute value, the significant feature is the relative magnitude of the central (zero frequency) peak and the side (coherent) peaks, which essentially reflects the underlying dynamics, say, being incoherent or partially coherent. (iii) The position of the coherent peak is around the Rabi frequency $\omega_{R}$. This is understandable, because it is the Rabi oscillation which causes the "coherent" oscillating behavior of the occupation probability. As we have pointed out, no quantum coherence is introduced and caused between the occupied and unoccupied dot states. However, the coherent flipping of the spin-up and spin-down states of the electron inside the quantum dot would induce the coherencelike oscillation of the occupation probability, with the same Rabi frequency, as shown in Fig. 4. The QPC can probe this shorttime oscillating behavior and results in the coherent peaks in the output noise spectrum. Precisely speaking, the peak po- 
sition does not locate exactly at the Rabi frequency. This can also be understood from the analytical result of the noise spectrum in the measurement of solid-state charge qubit ${ }^{21}$ by the QPC. However, the slight shift of the peak position to higher frequency with $\Gamma$ does not provide any interesting information.

In summary, we have presented a Monte Carlo simulation for the single realization of continuous detection of singleelectron spin. The measurement scheme, say, based on the spin-to-charge conversion and performing the electrical detection by a mesoscopic detector, is of high interest for quantum computing. The present study provides a theoretical description for the measured RTS result, ${ }^{8,9}$ which may represent an experimental breakthrough of single-electron spin detection in solid states. The predicted feature in the output noise spectrum reveals an interesting interplay of coherent oscillation and incoherent tunneling. It is possible that observation in experiment has the value of directly illustrating the single-electron quantum dynamics in different regimes, i.e., from (partial) quantum to classical.

\section{ACKNOWLEDGMENTS}

Support from the National Natural Science Foundation of China and the Research Grants Council of the Hong Kong Government are gratefully acknowledged.
${ }^{1}$ G. P. Berman, G. W. Brown, M. E. Hawley, and V. I. Tsifrinovich, Phys. Rev. Lett. 87, 097902 (2001).

${ }^{2}$ G. P. Berman, F. Borgonovi, G. Chapline, S. A. Gurvitz, P. C. Hammel, D. V. Pelekhov, A. Suter, and V. I. Tsifrinovich, J. Phys. A 36, 4417 (2003).

${ }^{3}$ Jinshuang Jin and Xin-Qi Li, Appl. Phys. Lett. 86, 143504 (2005).

${ }^{4}$ H. A. Engel, V. N. Golovach, D. Loss, L. M. K. Vandersypen, J. M. Elzerman, R. Hanson, and L. P. Kouwenhoven, Phys. Rev. Lett. 93, 106804 (2004).

${ }^{5}$ D. Gammon, A. L. Efros, T. A. Kennedy, M. Rosen, D. S. Katzer, D. Park, S.W. Brown, V. L. Korenev, and I.A. Merkulov, Phys. Rev. Lett. 86, 5176 (2001).

${ }^{6}$ F. Jelezko, T. Gaebel, I. Popa, A. Gruber, and J. Wrachtrup, e-print quant-ph/0309079 (unpublished).

${ }^{7}$ D. Rugar, R. Budakian, H. J. Mamin, and B. W. Chui, Nature (London) 430, 329 (2004).

${ }^{8}$ J. M. Elzerman, R. Hanson, L. H. Willems van Beveren, B. Witkamp, L. M. K. Vandersypen, and L. P. Kouwenhoven, Nature (London) 430, 431 (2004).

${ }^{9}$ M. Xiao, I. Martin, E. Yablonovitch, and H. W. Jiang, Nature (London) 430, 435 (2004).

${ }^{10}$ S. A. Gurvitz, Phys. Rev. B 56, 15215 (1997).

${ }^{11}$ X. Q. Li, W. K. Zhang, P. Cui, J. Shao, Z. Ma, and Y.J. Yan, Phys. Rev. B 69, 085315 (2004).

${ }^{12}$ X. Q. Li, J. Luo, Y. G. Yang, P. Cui, and Y. J. Yan, Phys. Rev. B 71, 205304 (2005).

${ }^{13}$ H. S. Goan, G. J. Milburn, H. M. Wiseman, and H. B. Sun, Phys. Rev. B 63, 125326 (2001).

${ }^{14}$ Here, based on Eq. (3), we have concluded that the QPC has no influence on the ensemble-averaged unconditional state evolution. From the following, Eq. (5) and the formulas above Eq. (8), the same conclusion can be arrived for the conditional state evolution, by noting that the incoherent tunneling between the quantum dot and the side reservoir would lead to an exclusive dot-occupation probability of either " 0 " or " 1 ."

${ }^{15}$ I. Martin, D. Mozyrsky, and H. W. Jiang, Phys. Rev. Lett. 90, 018301 (2003).

${ }^{16}$ M. O. Scully and M. S. Zubairy, Quantum Optics (Cambridge University Press, Cambridge, UK, 1997).

${ }^{17}$ H. M. Wiseman, Ph.D. thesis, University of Queensland, 1994 (unpublished); Phys. Rev. A 49, 2133 (1994).

${ }^{18}$ A. N. Korotkov, Phys. Rev. B 60, 5737 (1999).

${ }^{19}$ H. M. Wiseman and G. Milburn, Phys. Rev. A 47, 1652 (1993).

${ }^{20}$ Hsi-Sheng Goan and Gerard J. Milburn, Phys. Rev. B 64, 235307 (2001).

${ }^{21}$ X. Q. Li, P. Cui, and Y. J. Yan, Phys. Rev. Lett. 94, 066803 (2005). There, the setup of a charge qubit measured by a QPC was studied. Despite the same measurement principle in comparison with the one studied here, the electron dynamics in the quantum dot and the effect of the detector's back action on it are quite different. That is, for the setup studied in the present article, the QPC's back action does not induce transition between the occupied and unoccupied dot states, and the tunnel coupling with the side reservoir does not cause phase coherence between them. As a result, the ESR-induced coherent oscillating behavior of the occupation probability is relatively weak, which only results in the small coherent peak in the noise spectrum. However, the dependence of the peak position on the Rabi frequency $\omega_{R}$ and the dephasing rate $\Gamma$ should be qualitatively the same. 